\title{
Dark Matter versus MOND
}

\author{
T. R. Mongan \\ 84 Marin Avenue, Sausalito, California, USA \\ Email:tmongan@gmail.com
}

How to cite this paper: Mongan, T.R. (2017) Dark Matter versus MOND. Journal of Modern Physics, 8, 919-922. https://doi.org/10.4236/jmp.2017.86057

Received: April 27, 2017

Accepted: May 23, 2017

Published: May 26, 2017

Copyright $\odot 2017$ by author and Scientific Research Publishing Inc. This work is licensed under the Creative Commons Attribution International License (CC BY 4.0).

http://creativecommons.org/licenses/by/4.0/

\section{Open Access}

\begin{abstract}
Many physicists believe dark matter accounts for flat velocity curves in spiral galaxies and find further evidence for dark matter in observations of the colliding "bullet cluster" galaxies 1E0657-56. Others claim a modified law of gravity called MOND (MOdified Newtonian Dynamics) explains galactic velocity curves better than dark matter. Merritt recently argued for MOND (arXiv:1703.02389) by claiming dark matter models cannot account for the MOND acceleration threshold $a_{0} \approx 1.2 \times 10^{-8} \mathrm{~cm} / \mathrm{sec}^{2}$ and the

$\left(V_{\text {observed }} / V_{\text {Newtonian }}\right)$ relation. However, this note shows that the HLSS model involving dark matter accounts for both the MOND acceleration and the $\left(V_{\text {observed }} / V_{\text {Newtonian }}\right)$ relation. After this paper was accepted for publication, I learned that Man Ho Chan previously reached the same conclusion (arXiv:1310.6801) using a dark matter based analysis independent of the holographic approach used in this paper.
\end{abstract}

\section{Keywords}

Galactic Structure, Dark Matter, MOND (MOdified Newtonian Dynamics)

\section{Introduction}

Dark matter is generally believed to account for the approximately flat velocity curves characteristic of spiral galaxies. Observations of the colliding "bullet cluster" galaxies 1E0657-56 provide further evidence for the existence of dark matter. However, some physicists believe the observed flat velocity curves indicate the law of gravity must be modified at large distances according to MOdified Newtonian Dynamics (MOND). Recently Merritt [1] argued for MOND by claiming dark matter models cannot account for the acceleration threshold $a_{0} \approx 1.2 \times 10^{-8} \mathrm{~cm} / \mathrm{sec}^{2}$ and the $\left(V_{\text {observed }} / V_{\text {Newtonian }}\right)$ relation emerging from the MOND approach [2].

\section{Purpose}

The purpose of this paper is to evaluate the validity of Merritt's claim by 
considering a specific model based on dark matter, the holographic large scale structure (HLSS) model [3]. The HLSS model was developed within the $\Lambda$ CDM paradigm and employs the holographic principle based on thermodynamics and general relativity [4]. This note shows the HLSS model can account for both the MOND acceleration threshold and the $\left(V_{\text {observed }} / V_{\text {Newtonian }}\right)$ relation.

\section{Analysis}

In the HLSS model, galaxies with total mass $M_{g}$ inhabit spherical holographic screens with radius $R_{S}=\sqrt{\frac{M_{g}}{0.183 \mathrm{~g} / \mathrm{cm}^{2}}}$ if the Hubble constant $H_{0}=67.8 \mathrm{~km} \cdot \mathrm{sec}^{-1} \cdot \mathrm{Mpc}^{-1}$. The HLSS model considers galactic matter density distributions $\rho(r)=\frac{M_{g}}{4 \pi R_{S} r^{2}}$, where $r$ is the distance from the galactic center. The spherical isothermal halo of dark matter, with radius $R_{S}$ and mass $M_{D M}=0.84 M_{g}$, has density distribution $\rho_{D M}(r)=\frac{M_{D M}}{4 \pi R_{S} r^{2}}$ so the dark matter mass within radius $R$ is $\frac{R}{R_{S}} M_{D M}$. There is no singularity in the galactic matter density distribution $\rho(r)=\frac{M_{g}}{4 \pi R_{S} r^{2}}$ because mass inside a core volume of radius $R_{c}$ at the galactic center is concentrated in a central black hole with mass $M_{C B H}=\frac{R_{c}}{R_{S}} M_{g}$ [3]. Radial acceleration at radius $R$ due to dark matter is then $a_{D M}=\frac{G}{R^{2}}\left(\frac{R}{R_{s}}\right) M_{D M}$. At radii $R$ sufficiently distant from the galactic center that total baryonic mass of the galaxy $M_{B}=0.16 M_{g}$ can be treated as concentrated at the galactic center, Newtonian radial acceleration resulting from baryonic matter is $a_{B}=\frac{G M_{B}}{R^{2}}$. The radius $R_{\gamma}$ where $a_{D M}=a_{B}$ is found from

$$
\frac{G}{R_{\gamma}^{2}}\left(\frac{R_{\gamma}}{R_{S}}\right) M_{D M}=\frac{G M_{B}}{R_{\gamma}^{2}} .
$$

Since $M_{D M}=0.84 M_{g}$ and $M_{B}=0.16 M_{g}, R_{\gamma}=0.19 R_{S}$, and at that radius

$$
a_{D M}=a_{B}=a_{0}=5.4 \times 10^{-8} \mathrm{~cm} / \mathrm{sec}^{2}
$$

consistent with the MOND estimate $a_{0} \approx 1.2 \times 10^{-8} \mathrm{~cm} / \mathrm{sec}^{2}$.

Another indication that the MOND acceleration $a_{0} \approx 1.2 \times 10^{-8} \mathrm{~cm} / \mathrm{sec}^{2}$ is a natural scale in the dark matter based HLSS model involves the situation at the radius $R_{S}$ of the spherical holographic screen. Then the Newtonian assumption, that total galactic mass can be considered as concentrated at the galactic center, is certainly mathematically justified. There, the sum of radial acceleration from dark matter and radial acceleration from baryonic matter is

$$
a_{S}=\frac{G M_{D M}}{R_{S}^{2}}+\frac{G M_{B}}{R_{S}^{2}}=\frac{G}{R_{S}^{2}}\left(M_{D M}+M_{B}\right) .
$$


Using

$$
M_{D M}+M_{B}=M_{g}=0.183 R_{S}^{2}
$$

then yields

$$
a_{S}=0.183 G=1.2 \times 10^{-8} \mathrm{~cm} / \mathrm{sec}^{2}
$$

equal to the estimated MOND acceleration $a_{0} \approx 1.2 \times 10^{-8} \mathrm{~cm} / \mathrm{sec}^{2}$.

The tangential velocity $V$ at radius $R$ is related to radial acceleration $a_{r}$ by $V^{2}=R a_{r}$. So, the ratio ( $\left.V_{\text {observed }} / V_{\text {Newtonian }}\right)$ is approximately

$$
\left(\frac{V_{\text {observed }}}{V_{\text {Newtonian }}}\right)^{2} \approx \frac{R\left(a_{D M}+a_{B}\right)}{R a_{B}},
$$

resulting in

$$
\frac{V_{\text {observed }}}{V_{\text {Newtonian }}}=\sqrt{1+\frac{a_{D M}}{a_{B}}}=\sqrt{1+\frac{R M_{D M}}{R_{S} M_{B}}} .
$$

Then, when $R \ll 0.19 R_{s}$,

$$
\frac{V_{\text {observed }}}{V_{\text {Newtonian }}} \approx 1
$$

as noted by Merritt [1]. Next, using

$$
\frac{a_{0}}{a_{B}}=\frac{M_{D M}}{0.19 M_{B}}\left(\frac{R}{R_{S}}\right)^{2}
$$

and

$$
\frac{R}{R_{S}}=\sqrt{\frac{0.19 M_{B}}{M_{D M}}} \sqrt{\frac{a_{0}}{a_{B}}}
$$

results in

$$
\frac{V_{\text {observed }}}{V_{\text {Newtonian }}}=\sqrt{1+\frac{R M_{D M}}{R_{S} M_{B}}}=\sqrt{1+\sqrt{\frac{0.19 M_{D M}}{M_{B}}} \sqrt{\frac{a_{o}}{a_{B}}}} .
$$

When $a_{B} \ll a_{0}, \frac{a_{O}}{a_{B}} \gg 1$ and

$$
\frac{V_{\text {observed }}}{V_{\text {Newtonian }}}=\sqrt{1+0.998 \sqrt{\frac{a_{o}}{a_{B}}}} \approx\left(\frac{a_{0}}{a_{B}}\right)^{\frac{1}{4}},
$$

again as noted by Merritt [1]. Since $\left(\frac{V_{\text {observed }}}{V_{\text {Newtonian }}}\right)^{4}=\frac{a_{0}}{a_{B}}$ when $a_{B} \ll a_{0}$, using

$$
\begin{aligned}
a_{B}=\frac{G M_{B}}{R^{2}} \text { and } V_{\text {Newtonian }} & =\sqrt{\frac{G M_{B}}{R^{2}}} \text { gives } \\
V_{\text {observed }}^{4} & =\left(\frac{G M_{B}}{R^{2}}\right)^{2}\left(\frac{R^{2}}{G M_{B}}\right) a_{0}=G M_{B} a_{0}
\end{aligned}
$$

also known as the baryonic Tully-Fisher relation.

Finally, if the Hubble constant $H_{0}=67.8 \mathrm{~km} \cdot \mathrm{sec}^{-1} \cdot \mathrm{Mpc}^{-1}$, the cosmological constant $\Lambda=1.12 \times 10^{-56} \mathrm{~cm}^{-2}$, and the accelerations $c H_{0}=6.6 \times 10^{-8} \mathrm{~cm} / \mathrm{sec}^{2}$ 
and $c^{2} \sqrt{\frac{\Lambda}{3}}=5.5 \times 10^{-8} \mathrm{~cm} / \mathrm{sec}^{2}$ are both consistent with the acceleration

$a_{0}=5.4 \times 10^{-8} \mathrm{~cm} / \mathrm{sec}^{2}$ estimated above.

\section{Conclusion}

Contrary to Merritt's claim [1], this note demonstrates that the HLSS model [3], based on dark matter, can account for the MOND acceleration threshold, the $\left(V_{\text {observed }} / V_{\text {Newtonian }}\right)$ relation, and the baryonic Tully-Fisher relation. After this paper was accepted for publication, I learned that Man Ho Chan previously reached the same conclusion [5] using a dark matter based analysis independent of the holographic approach used in this paper.

\section{Acknowledgements}

I thank the reviewer for important suggestions about how to improve the presentation of these results.

\section{References}

[1] Merritt, D. Cosmology and Convention. arXiv:1703.02389

[2] Famaey, B. and Mc Gaugh, S. (2012) Living Reviews in Relativity, 15, 10. arXiv:1112:3960

[3] Mongan, T.R. (2011) JMP, 2, 1544, and (2013) JMP, 4, 50.

[4] Bousso, R. (2002) Reviews of Modern Physics, 74, 825. https://doi.org/10.1103/RevModPhys.74.825

[5] Chan, M.H. (2013) Physical Review D, 88, 103501. arXiv:1310.6801 https://doi.org/10.1103/PhysRevD.88.103501

\section{Submit or recommend next manuscript to SCIRP and we will provide best service for you:}

Accepting pre-submission inquiries through Email, Facebook, LinkedIn, Twitter, etc. A wide selection of journals (inclusive of 9 subjects, more than 200 journals)

Providing 24-hour high-quality service

User-friendly online submission system

Fair and swift peer-review system

Efficient typesetting and proofreading procedure

Display of the result of downloads and visits, as well as the number of cited articles

Maximum dissemination of your research work

Submit your manuscript at: http://papersubmission.scirp.org/

Or contact jmp@scirp.org 